\title{
An Multi-Mini-Partition Intra Block Copying for Screen Content Coding
}

\author{
LiPing $\mathrm{ZHAO}^{1, \mathrm{a}^{*}}$, Tao LIN ${ }^{2, \mathrm{~b}}$
}

${ }^{1}$ College of Mathematics, Physics and Information Engineering, JiaXing University, JiaXing, China

${ }^{2}$ VLSI Lab,Tongji University, Shanghai, China

aemail:zhaoliping_jian@126.com, bemail: lintao@tongji.edu.cn

Keywords: screen content coding, Intra block copying, mini-partition matching

Abstract. In this paper, an multi-mini-partition intra block copying (MMPIBC) coding technique for screen contents is presented. In MMPIBC coder, a Coding Unit(CU) with size $8 \times 8$ is firstly broken into multiple mini-partitions. Each mini-partition of CU separately select the displacement vector(DV) with the same search range. To further decrease the RD-cost of MMPIBC mode, DV predictor and pseudo three planar search techniques are applied to get more coding gain. Experiments show that for some types of screen contents, the average Bit-rate saving of MMPIBC achieves up to $14.9 \%$.

\section{Introduction}

Screen content coding (SCC) is a key technology for some emerging popular applications such as WIFI display, cloud computing, cloud-mobile computing, and remote desktop[1],[2]. The challenging requirement in SCC is to achieve both ultra-high visually lossless quality and ultra-high compression ratio up to 300:1 3000:1. In recent years, SCC has attracted increasing attention of researchers from both academia and industry[3]-[8]. The Joint Collaborative Team on Video Coding (JCT-VC) working on High Efficiency Video Coding (HEVC) standardization has also established SCC ad hoc groups to progress SCC technology and standardization[4].

Typical computer screens seen in daily use are often rich in small and sharp bitmap structures such as text, menu, icon, button, slide-bar, and grid. There are usually many similar or identical patterns in a screen picture. A full page of English text consists of only 52 capital and small letters, which all consist of even fewer numbers of basic strokes. Most Asian texts also consist of 5-10 basic strokes.

LZMA-based schemes[5],[6] achieve high efficiency for screen content coding because there are a lot of repeating patterns which can be jointly compressed by LZMA-based scheme. However, the 1-D dictionary coding scheme treat the input signal as a 1-D signal which significantly breaks the structure of 2-D patterns in screen contents. Intra block copying[7][8] used in traditional hybrid coding within a picture has been proved to be a efficient way to code similar or identical pattern. For the coding units (CUs) using intra block copying, the prediction block is obtained from the reconstructed region. Then, the displacement vectors(DVs) and residual are coded.

However, IBC with the smallest size $8 x 8$ will lead to large residual signal . In this paper, we present an MMPIBC coding technique for screen contents. In MMPIBC coder, a Coding Unit(CU) with size $8 \times 8$ is broken into multiple mini-partitions. Each mini block separately select the displacement vector(DV) with the same search range. Then the multi-DV and the residual signal are entropy-coded into the bitstream buffer. At the same time, each CU is also coded by traditional IBC matching based hybrid-coder. Finally, the coder with the minimal rate-distortion (RD) cost is selected as the final coder to compress the $\mathrm{CU}$ and the corresponding bitstream is output to the final bitstream.

\section{MMPIBC Coding System}

\section{encoder and eecoder architecture of MMPIBC}

a) shows the encoder and decoder architecture of MMPIBC. In the encoder, an MMPIBC unit is added in parallel with the prediction unit in a traditional hybrid coder(in ). An input Coding Unit (CU) with size $8 \times 8$ is fed to both MMPIBC unit IBC unit and prediction unit. A mode Index flag indicates whether the coding unit is MMPIBC or IBC type. In MMPIBC coding Unit, CU with size $8 \times 8$ is firstly 
broken into 16 mini-partitions with size $4 \times 1$. Each mini-partition separately selects the best displacement vector(DV). Multi-DV and DV are obtained from one IBC search in order to save coding time. The mode information such as multi-DV 、 DV or intra/inter mode information are entropy-coded. Then, pixel residuals from both MMPIBC unit IBC unit and prediction unit separately go through the rest of the traditional hybrid coding steps. Although, multi-DV may increase the mode information(also called mode decision data) entropy bits, but the bits for entropy the pixel residuals will decrease gradually because of mini-partition pattern copying. Finally, MMPIBC-based coding or IBC-based coding or traditional prediction-based coding is selected to code the CU based on $\mathrm{RD}$ cost evaluation and the corresponding bitstream is put into the MMPIBC output bitstream.

In the decoder, the input MMPIBC bitstream is first parsed to separate MMPIBC bitstream from IBC-based bitstream and prediction-based bitstream on a CU-by-CU basis. Then, the prediction-based bitstream is decoded by the traditional hybrid coding steps to get a reconstructed CU. On the other hand, the MMPIBC bitstream and IBC bitsteam is decoded by MMPIBC or IBC steps determined by the mode index flag. Then prediction signals are reconstructed by applying motion compensation (MC) using the multi-DV or DV .The MMPIBC or IBC residuals, if any, are added to get a reconstructed CU.

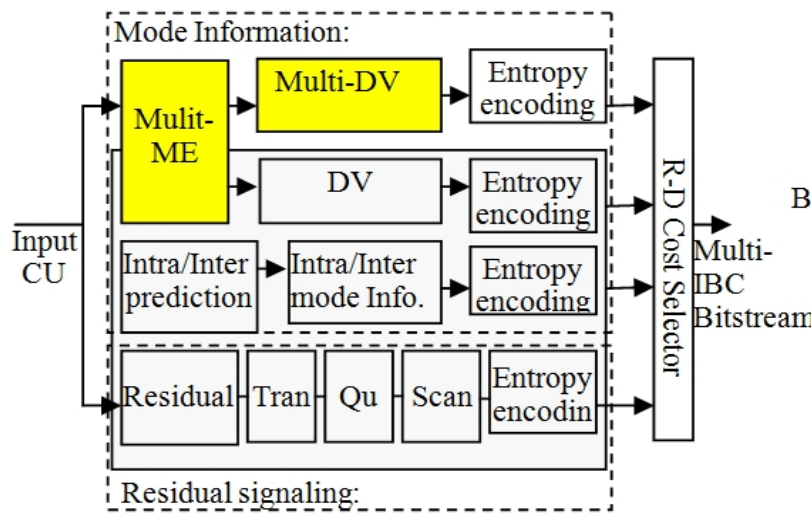

a) Encoder

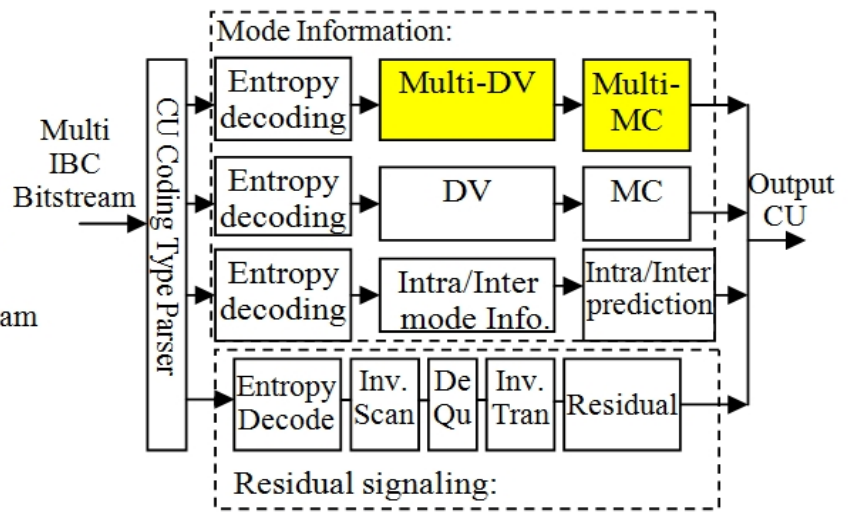

b) Decoder

Fig1. MMPIBC coding architecture.

\section{horizontal-scanned MMPIBC search}

An example of CU m with size $8 \times 8$ coded by horizontal scanned MMPIBC scheme is shown in 0 . The LCU size is $64 \times 64$. The searching area (previously coded and reconstructed pixels) is treated as the same as in IBC coding scheme . CU m is firstly broken into 16 mini-partition with size $4 \times 1$. From the horizontal scanned order, each mini-partition will find the best DV in the search area. 0 shows the first six DVs of mini-partition for CU m:

1) The DV of the 1 st mini partiton is $(-8,48)$;

2) The DV of the 2nd mini partiton is $(48,32)$;

3) The DVs of 3rd 、4rd 、 5rd 、6rd mini partiton are the same $(0,48)$.

The search area used in this paper is the current CTU and the left CTU . In order to save the coding time, multi DVs or DV are obtained from one intra block search in motion estimation. Then the multi DVs are fed to the MC process to get the residual signals. The multi DVs and the residual signals finally are entropy-coded.

In the conventional HEVC, mode decision is determined by RD cost(shown in Eq. 1).

$$
\mathrm{RDC}=\mathrm{D}+\lambda \mathrm{R}
$$

Take lossless coding for example, the prediction error(D) equals 0 and $\lambda$ equals $1(\mathrm{QP}=0)$. As shown in Eq.2, the RD cost is determined by the total bits which entropy-coded into the bitstream buffer.

$$
\mathrm{R}=\mathrm{Bits}(\text { residual)+Bits(multi-DV) }
$$

Obviously, the bits used for multi-DV entropy of MMPIBC are much more than that of IBC, because the DV number of MMPIBC is 16 times of IBC. However, if the mini-partition block copying led to smaller residual data in MMPIBC, the bits used for residual signals entropy can decrease gradually, then the coding gain is obtained. 


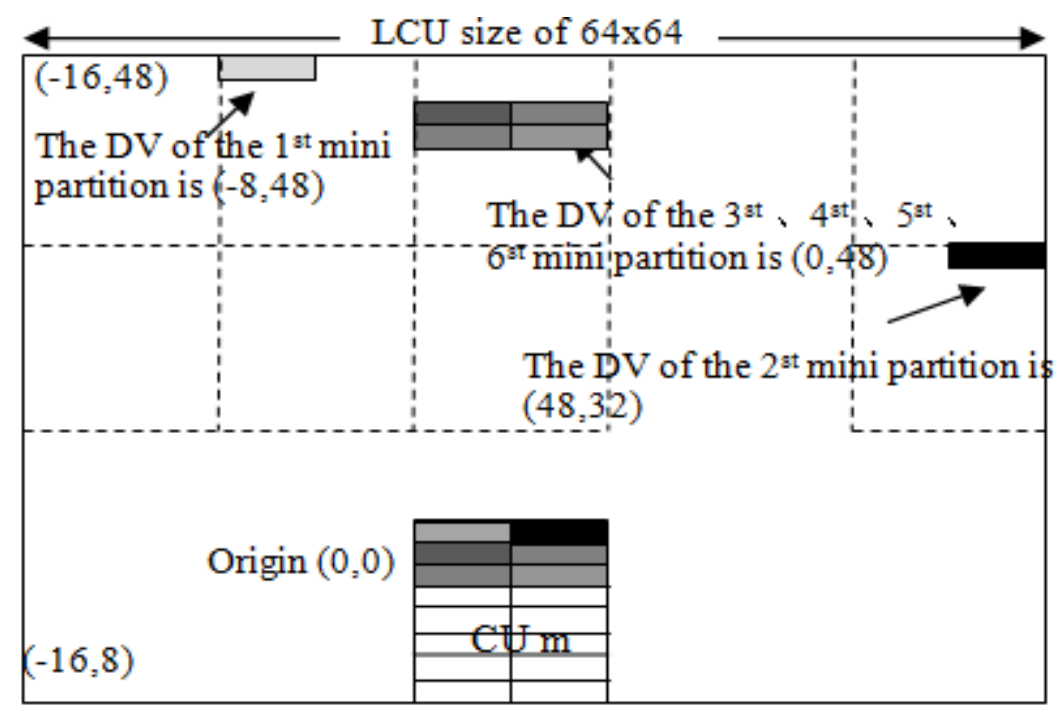

Fig2. horizontal scanned MMPIBC with size 8x8.

\section{MMPIBC-oriented techniques}

\section{pseudo three plannar search}

As shown in Table 1, in order to find the best DV for each mini partition, firstly find the best fourth DV from the Y components pattern search. Then, for each DV in the best fourth DV, get the sum of abstract distortion(SAD) of UV components. The total SAD is obtained by adding the SAD of Y、 U、 V components. The DV with the minimum total SAD of the best fourth DV is the final DV.

Table 1. pseudo three palanar search algorithm

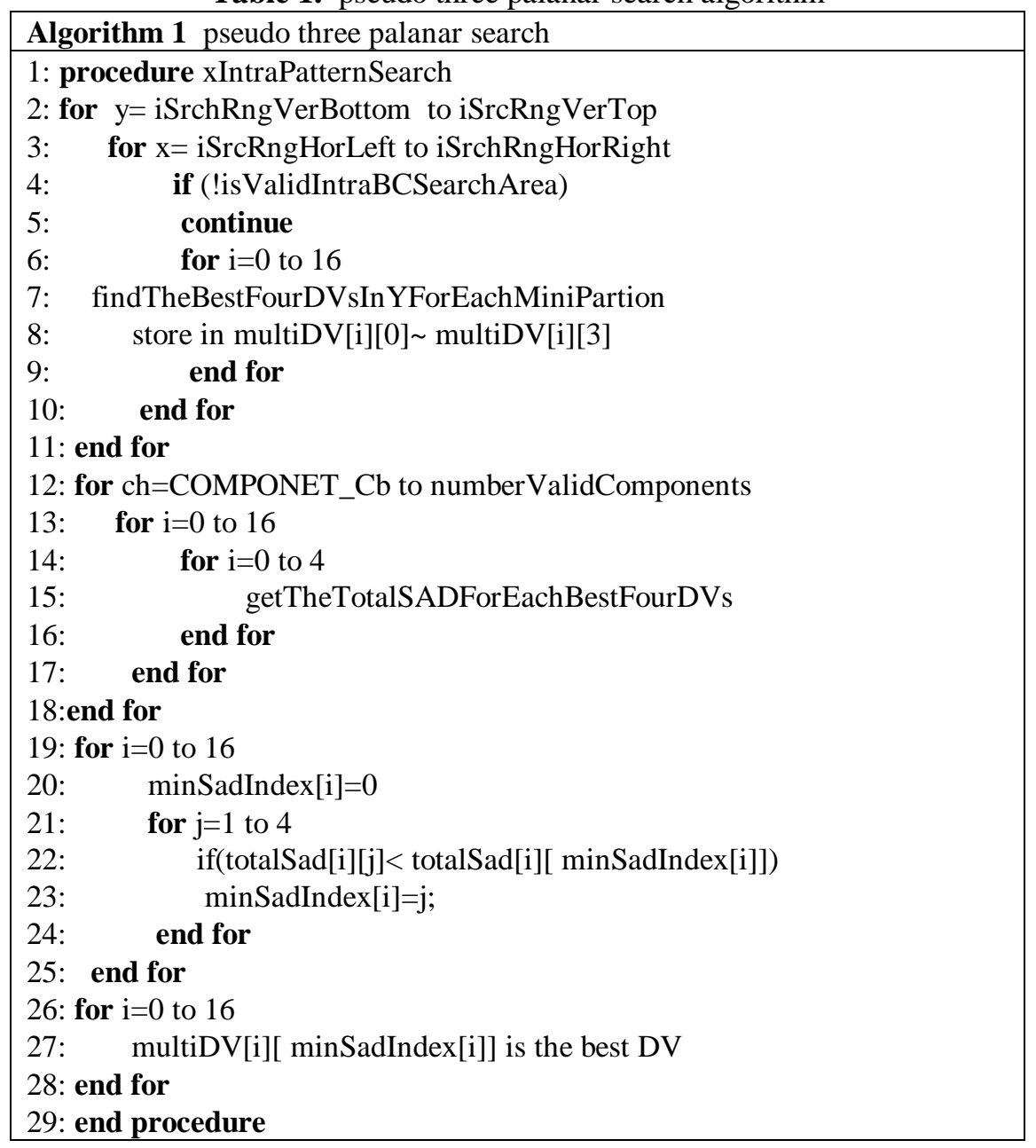




\section{DV predictor}

Notice that the DVs of $3^{\text {rd }} 、 4^{\text {rd }} 、 5^{\text {rd }} 、 6^{\text {rd }}$ mini partition are the same(see in Fig. 2) . The former two DVs hold their raw DV value, and the following DV selects its former two DV as predictor and the resulting displacement vector difference (DVD) is coded. For easy understanding of the scheme, Table 2 shows an example of raw 16 DV value and their predicted 16 DV value. Obviously, the DVD after prediction contains many DV of zero value, which costs the smallest bits of DV entropy-coded.

Table 2. DV predictor scheme

\begin{tabular}{|c|c|c|c|}
\hline \multicolumn{2}{|c|}{ DV } & \multicolumn{2}{c|}{ DV } \\
\hline $\mathrm{x}$ & $\mathrm{y}$ & $\mathrm{x}$ & $\mathrm{y}$ \\
\hline-71 & 21 & -15 & 0 \\
\hline 0 & -3 & 0 & -3 \\
\hline-71 & 21 & -71 & 21 \\
\hline-71 & 21 & -71 & 21 \\
\hline-15 & 0 & -15 & 0 \\
\hline-15 & 0 & -15 & 0 \\
\hline-15 & 0 & -15 & 0 \\
\hline-15 & 0 & -15 & 0 \\
\hline
\end{tabular}

a)raw $16 \mathrm{DV}$

\begin{tabular}{|c|c|c|c|}
\hline \multicolumn{2}{|c|}{ DV } & \multicolumn{2}{c|}{ DV } \\
\hline $\mathrm{x}$ & $\mathrm{y}$ & $\mathrm{x}$ & $\mathrm{y}$ \\
\hline-71 & 21 & -15 & 0 \\
\hline 71 & -24 & 15 & -3 \\
\hline-71 & 24 & -71 & 24 \\
\hline 0 & 0 & 0 & 0 \\
\hline 0 & 0 & 0 & 0 \\
\hline-56 & -21 & -56 & -21 \\
\hline 0 & 0 & 0 & 0 \\
\hline 0 & 0 & 0 & 0 \\
\hline
\end{tabular}

b)predicted $16 \mathrm{DV}$

\section{Experimental Results}

In this section, experimental results are presented for the proposed MMPIBC methods. The methods are implemented in HM12.1_RExt5.1[9] and simulated under the RCE3 common test conditions[10]. The benchmark testing data are JCT-VC SCC ad hoc group common tests[10]. They represent quite a few different typical screen content scenarios such as a variety of hardware and software design engineering, programming, video conferencing with document sharing, web browsing, computer graphics rendering, map navigating and address direction searching, and document editing. All the data are captured in raw 24-bit RGB format.

The performance is compared to the anchor for lossless configuration in terms of Bit-rate saving. In the experiments, other conditions used in the experiment:1) Configuration: All-intra. 2) Four algorithms: MMPIBC(shorted for Method1)、MMPIBC+ pseudo three planar search(shorted for Method2) 、 MMPIBC+DV predictor(shorted for Method3) and MMPIBC+ DV predictor + pseudo three planar search(shorted for Method4). 3) The search area is the current CTU and the left CTU . Compared to the HEVC base hybrid coder, results of Method1、Method2、Method3 and Method4 are shown in Table 3 for lossless.

Table 3. results comparison between four algorithms

\begin{tabular}{lllll}
\hline Bit-rate saving (Average) & Method 1 & Method 2 & Method 3 & Method 4 \\
\hline SC RGB 444 & $-4.5 \%$ & $-5.6 \%$ & $\mathbf{- 8 . 9 \%}$ & $\mathbf{- 1 0 . 6 \%}$ \\
Animation RGB 444 & $0.0 \%$ & $0.0 \%$ & $0.0 \%$ & $0.0 \%$ \\
SC YUV 444 & $-4.8 \%$ & $-5.5 \%$ & $\mathbf{- 9 . 5 \%}$ & $\mathbf{- 1 0 . 8 \%}$ \\
Animation YUV 444 & $0.0 \%$ & $0.0 \%$ & $0.0 \%$ & $0.0 \%$ \\
SC GBR 444 Optional & $-3.1 \%$ & $-4.1 \%$ & $\mathbf{- 9 . 7 \%}$ & $\mathbf{- 1 1 . 6 \%}$ \\
SC YUV 444 Optional & $-5.9 \%$ & $-6.2 \%$ & $\mathbf{- 1 4 . 4 \%}$ & $\mathbf{- 1 4 . 9 \%}$ \\
Enc Time[\%] & $183.04 \%$ & $184.01 \%$ & $192.62 \%$ & $206.35 \%$ \\
Dec Time[\%] & $97.00 \%$ & $97.26 \%$ & $97.91 \%$ & $96.21 \%$ \\
\hline
\end{tabular}

From the experiment data, we have the following findings:

1) In method 4, for the SC RGB (YUV)444 and the SC RGB (YUV)444 Optional, the average coding gain (BD-rate reduction) of the MMPIBC coder over HEVC base coder is more than $10 \%$.

2) DV predictor scheme is very efficient, for SC YUV 444 Optional ,the average coding gain of Method 3 over Method 1 is $8.5 \%$ with little encoding time increased. 
3) The improvement (gain) of the MMPIBC technique is heavily dependent on the contents. For some screen contents, the gain can be as high as more than $19.73 \%$, while for some other screen contents, there is no gain.

\section{Conclusions}

This paper presents the MMPIBC coding technique for SCC and in particular, a CU with size 8x8 horizontal scanned MMPIBC is proposed. The MMPIBC bitstream is a combination of traditional hybrid coding bitstream and MMPIBC coding bitstream mixed on an CU-by-CU basis. DV predictor scheme and pseudo three planner search scheme are also proposed to get more coding gain of MMPIBC. Experiments show that the MMPIBC coding achieves much better BD-rate performance than hybrid coder for screen contents. Future work includes 1) decreasing the bite rate by analyzing the characteristic of multi DVs; 2) finding a good balance between the compression ratio and the DV number. 3)decreasing the encode time by fast motion estimation.

\section{Acknowledgement}

This work is supported by the Key issues of Scientific Research of JiaXing University (No.70112027BL), the Science and Technology Project of JiaXing(No. 2014AY11004), Natural Science Foundation of Zhejiang Province(No.LY15F020039), National Natural Science Foundation of China (No.61201226, No.61271096), the Specialized Research Fund for the Doctoral Program of Higher Education (No.20130072110054).

\section{References}

[1] Yan Lu, Shipeng Li and Huifeng Shen, "Virtualized Screen: A Third Element for Cloud_Mobile Convergence," IEEE Multimedia, Vol. 18, no. 2, pp. 4-11, Apr. 2011.

[2] Tao Lin, Kailun Zhou, and Shuhui Wang. "Cloudlet-Screen Computing: A Client-server Architecture with Top Graphics Performance," International Journal of Ad Hoc and Ubiquitous Computing, Vol. 13, no. 2, pp. 96-108, June 2013.

[3] M. Zhou, W. Gao, M. Jiang, H. Yu, "HEVC Lossless Coding and Improvements," IEEE Transactions on Circuits and Systems for Video Technology, Vol. 22 , no. 12, pp. 1839-1843, Dec. 2012.

[4] ISO/IEC JTC1/SC29/WG11 and ITU-T Q6/16. Joint call for proposals for coding of screen content. ISO/IEC JTC 1/SC 29/WG 11 (MPEG) Document N14175, San Jose, 2014.

[5] T. Lin, P. Zhang, S. Wang, K. Zhou, X. Chen, "Mixed Chroma Sampling-rate High Efficiency Video Coding for Full-chroma Screen Content," IEEE Transactions on Circuits and Systems for Video Technology, Vol. 23, no. 1, pp. 173-185, Jan. 2013.

[6] Tao Lin, Shuhui Wang, Peijun Zhang, and Kailun Zhou, "P2M based dual-coder extension of HEVC,” JCT-VC Doc JCTVC-L0303, Jan. 2013.

[7] Do-Kyoung Kwon, Madhukar Budagavi, "Intra motion compensation with variable length intra MV coding”, JCT-VC Doc JCTVC- N0206, July. 2013.

[8] Chao Pang, Joel Sole, Liwei Guo, Marta Karczewicz, Rajan Joshi, "Intra Motion Compensation with 2-D MVs" ,JCT-VC Doc JCTVC- N0256, July. 2013.

[9] https://hevc.hhi.fraunhofer.de/svn/svn_HEVCSoftware/tags/HM-12.1+RExt-5.1/

[10] A. Saxena, D. Kwon, M. Naccari, and C. Pang, "HEVC Range Extensions Core Experiment 3 (RCE3): Intra Prediction Techniques”, JCTVC-N1123, Vienna, AT, August 2013 